\title{
CYP1A1-Mediated Intramolecular Rearrangement of Aminoazepane in GDC-0339 ${ }^{\text {[ }}$
}

\author{
Ryan H. Takahashi, Xiaojing Wang, Nathaniel L. Segraves, ${ }^{1}$ Jing Wang, Jae H. Chang, \\ S. Cyrus Khojasteh, and Shuguang Ma
}

Departments of Drug Metabolism and Pharmacokinetics (R.H.T., J.W., J.H.C., S.C.K., S.M.), Discovery Chemistry (X.W.), and Small Molecule Pharmaceutical Sciences (N.L.S.), Genentech, Inc., South San Francisco, California

Received May 16, 2017; accepted August 7, 2017

\begin{abstract}
GDC-0339 is a novel small molecule pan-Pim kinase inhibitor that was discovered as a potential treatment of multiple myeloma. During the in vitro and in vivo metabolite profiling of GDC-0339, a metabolite was detected that had the same elemental composition as the parent but was distinct with respect to its chromatographic separation and mass spectrometric fragmentation pattern. High resolution tandem mass spectrometry data indicated the metabolite was modified at the aminoazepane moiety. The structure was solved by nuclear magnetic resonance analysis of the isolated metabolite and further confirmed by comparing it to a synthetic standard. These results indicated that the metabolite was formed by an intramolecular amine replacement reaction with the primary amine forming a new attachment to pyrazole without any change in stereochemistry. In vitro experiments showed cytochrome P450s catalyzed the
\end{abstract}

reaction and demonstrated high isoform selectivity by CYP1A1. Results from kinetic experiments showed that the CYP1A1mediated rearrangement of GDC-0339 was an efficient reaction with apparent turnover number $\left(\mathrm{k}_{\mathrm{cat}}\right)$ and Michaelis constant $\left(\mathrm{K}_{\mathrm{m}}\right)$ of 8.4 minutes $^{-1}$ and $0.6 \mu \mathrm{M}$, respectively. The binding of GDC-0339 to the cytochrome $\mathrm{P} 450$ active site was examined by characterizing the direct inhibition of CYP1A1-mediated phenacetin O-deethylation, and GDC-0339 was a potent competitive inhibitor with $\mathrm{K}_{\mathrm{i}}$ of $0.9 \mu \mathrm{M}$. This high affinity binding was unexpected given a narrow active site for CYP1A1 and GDC-0339 does not conform structurally to known CYP1A1 substrates, which are mostly polyaromatic planar molecules. Further, we explored some of the structural requirements for the rearrangement reaction and identified several analogs to GDC-0339 that undergo this biotransformation.

\section{Introduction}

Cytochrome P450s (P450s) catalyze a wide diversity of biotransformation reactions, though most frequently they are involved with oxidative reactions. Rearrangement reactions catalyzed by $\mathrm{P} 450$ s have been observed but are relatively rare (Guengerich and Munro, 2013). In most instances, the rearrangement is initiated by an oxidation step catalyzed by $\mathrm{P} 450$. Thus, the first metabolic step will introduce an oxygen atom that will identify the site modified by P450 and this can aid in rationalizing the mechanism for the reaction. Rearrangements involving a P450-intermediate complex, on the other hand, can be more challenging to rationalize. The product may differ from the parent only in chemical connectivity without the introduction or removal of any atoms that would indicate the nature and site for the initiating step of modifying the substrate. Some intriguing examples that have been mechanistically studied are the ring expansion of 1-phenylcyclobutylamine (Bondon et al., 1989) and the conversion of 7-dehydrocholesterol to

\footnotetext{
${ }^{1}$ Current affiliation: Theravance, Inc., South San Francisco, California. https://doi.org/10.1124/dmd.117.076786.

SThis article has supplemental material available at dmd.aspetjournals.org.
}

7-ketocholesterol (Shinkyo et al., 2011). Other key studies are described in the aforementioned review (Guengerich and Munro, 2013).

GDC-0339 (5-amino- $N$-(5-((4R,5R)-4-amino-5-fluoroazepan-1-yl)1-methyl-1H-pyrazol-4-yl)-2-(2,6-difluorophenyl)thiazole-4-carboxamide, Fig. 1) is a potent and orally bioavailable small molecule pan-Pim kinase inhibitor that was identified as a clinical candidate (Hodges et al., 2013). Inhibition of Pim kinases by either a single agent or in combination with standard or novel treatments is expected to be of clinical benefit in treating multiple myeloma (Keane et al., 2015). In vitro and in vivo metabolite profiling experiments of GDC-0339 revealed a metabolite that mass spectral data indicated to be a rearrangement product. Though it was observed with the same protonated molecular ion as the parent compound, it was chromatographically resolved and had a mass spectrometric fragmentation pattern unique from GDC-0339. The metabolite was of particular interest because there was no net oxidation-reduction from substrate to product, even though its formation was catalyzed by P450 enzymes and required external reducing equivalents (NADPHdependent formation).

In this report, we describe the experiments that characterized and solved the chemical structure of G-1025, the rearrangement product of GDC-0339. Given the unique nature of this rearrangement, we investigated it to identify the responsible P450 isoform, explored

ABBREVIATIONS: ABT, 1-aminobenzotriazole; G-1025, (5-amino-2-(2,6-difluorophenyl)- $N$-(5-((5-fluoroazepan-4-yl)amino)-1-methyl-1H-pyrazol4-yl)thiazole-4-carboxamide; GDC-0339, 5-amino- $N$-(5-((4R,5R)-4-amino-5-fluoroazepan-1-yl)-1-methyl-1H-pyrazol-4-yl)-2-(2,6-difluorophenyl)thiazole-4-carboxamide; $\mathrm{k}_{\mathrm{cat}}$, the turnover number; $\mathrm{K}_{\mathrm{m}}$, the Michaelis constant; LC, liquid chromatography; MS, mass spectrometry; MS/MS, tandem mass spectrometry; $\mathrm{m} / \mathrm{z}$, mass-to-charge ratio; NMR, nuclear magnetic resonance; P450, cytochrome P450. 


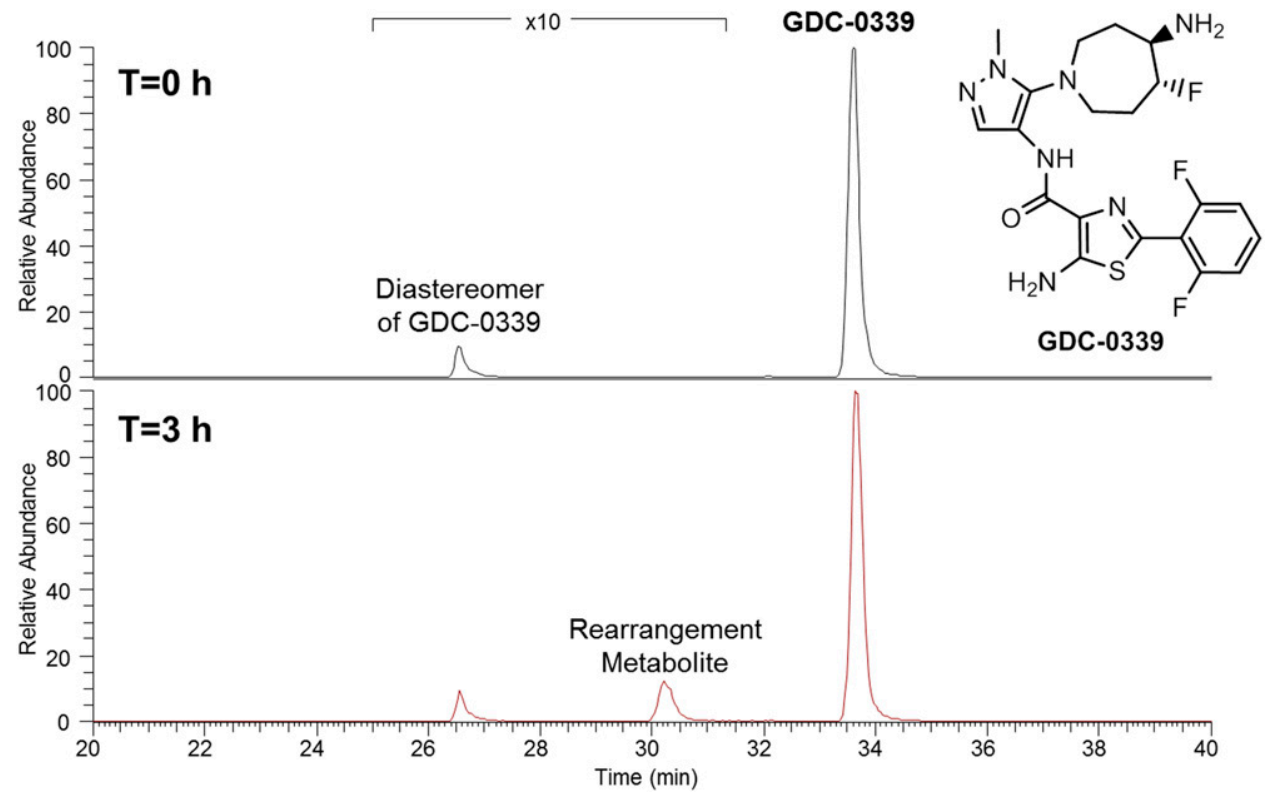

Fig. 1. Extracted ion chromatograms for human hepatocyte incubations of GDC-0339 at 0 and 3 hours. GDC-0339 (full structure shown) and its isomeric analytes were observed with protonated molecular ions within $5 \mathrm{ppm}$ of $\mathrm{m} / \mathrm{z}$ 466.1631. The rearrangement metabolite observed at retention time $\sim 30$ minutes was formed during the hepatocyte incubation.

the structural requirements for the substrate of this reaction, and proposed a mechanism that explained the intramolecular rearrangement.

\section{Materials and Methods}

\section{Materials}

GDC-0339 and a chemical standard of its metabolite, G-1025, were synthesized at Genentech, Inc. (South San Francisco, CA). Cryopreserved hepatocytes from male Sprague-Dawley rats and mixed sex (pool of 10 donors) humans were from Bioreclamation, Inc. (Westbury, NY). Pooled male rat and mixed-sex human (pool of 200 donors) liver microsomes and Supersomes containing cDNA-baculovirus-insect cell-expressed cytochromes P450 (CYP1A1, 1A2, 2B6, 2C8, 2C9, 2C18, 2C19, 2D6, 2E1, 3A4, and 3A5) were obtained from Corning (Woburn, MA). Liver microsomes from $\beta$-naphthoflavone-, dexamethasone-, and vehicle- (corn oil) pretreated rats were purchased from Xenotech (Lenexa, KS). Acetaminophen, $\alpha$-naphthoflavone, phenacetin, and propranolol (used as an analytical internal standard) were purchased from Sigma-Aldrich (St. Louis, MO). All other chemicals were purchased from vendors at the highest purity that was available.

\section{Metabolite Profiling and Comparison with Synthetic Standards}

In vitro (liver microsomes and hepatocyte incubations) and in vivo (rat urine) samples from GDC-0339 studies were profiled with liquid chromatography (LC)high resolution mass spectrometry (MS) experiments. The synthetic standards of G-1025 and its cis amino-fluoroazepane epimer (Table 1) were mixed with GDC0339-dosed rat urine to confirm coelution and the match of MS data to the in vivo metabolite. In earlier metabolite structure elucidation experiments, the desfluoro analog of GDC-0339 was incubated with liver microsomes to generate its rearrangement product. Mixing experiments of synthetic standards of proposed rearrangement products (Table 1) with the in vitro sample were completed to compare coelution and MS data. For profiling experiments, samples were analyzed using an LTQ-Orbitrap Velos mass spectrometer (Thermo Scientific, San Jose, CA) equipped with an Accela ultra performance liquid chromatography system comprised of a solvent pump with built-in degasser, temperaturecontrolled autosampler, and photodiode array detector (Thermo Scientific). The samples were chromatographed using a Kinetex $2.6 \mu \mathrm{m}$, XB-C18 $2.1 \times 100 \mathrm{~mm}$ (Phenomenex, Torrance, CA) column with a solvent gradient of $0.1 \%$ formic acid in water and methanol. High resolution full scan [mass-to-charge ratio $(\mathrm{m} / \mathrm{z}) 200$ $1000]$ and tandem mass spectrometry (MS/MS) experiments were completed at mass resolutions of 30,000 and 7500, respectively. The MS/MS product ion scans were obtained with data-dependent acquisitions using collision-induced dissociation with a normalized energy of $35 \%$. Additional instrument parameters are described in Supplemental Table 1.

The same profiling method was used to assess whether structural analogs of GDC-0339 underwent the rearrangement reaction. A rearrangement product was positively identified if an analyte with the same protonated molecular ion as the starting compound had formed during in vitro experiments. This rearrangement product was verified by production of abundant MS/MS product ion at $m / z, 351$, which was identified in the current work to be diagnostic for G-1025 and its analogs. No chemical standards were synthesized to confirm structures or quantity these rearrangement products.

\section{Metabolite Isolation and Nuclear Magnetic Resonance}

The rearrangement product was isolated from urine collected from male Sprague-Dawley rats $(n=3)$ and subjected to nuclear magnetic resonance (NMR) analysis for structure determination. Each rat received two doses at $300 \mathrm{mg} / \mathrm{kg}$ of GDC- 0339 by oral gavage at 0 and 8 hours. The oral dose of GDC-0339 was administered as a suspension in $0.5 \%$ methylcellulose and $1 \%$ Tween 80 . Urine from all animals out to 48 hours was pooled and concentrated under vacuum. Solids were removed from the sample by centrifugation and the supernatant was applied to a preparative scale C18 column for chromatography using a water/ acetonitrile gradient, with the eluents collected in fractions triggered by changes in the UV signal trace. The fractions that contained the rearrangement product were lyophilized to completely remove solvents. The isolated metabolite $(\sim 1 \mathrm{mg})$ was solubilized in $\mathrm{CD}_{3} \mathrm{OD}(99.8 \% \mathrm{D})$ with tetramethylsilane ( $0.05 \%$ by volume) as an internal chemical shift reference standard (Cambridge Isotope Laboratories, Inc., Tewksbury, MA). The sample was then transferred to a 5-mm NMR tube (535PP-7; Wilmad-LabGlass, Vineland, NJ), which was then purged with nitrogen and sealed. NMR measurements were performed on an Avance 3, 600-MHz spectrometer equipped with a 5-mm TXI CryoProbe (Bruker Daltonics, Billerica, MA). The sample temperature was maintained at $28^{\circ} \mathrm{C}$ for all data collections, and spectra were acquired using standard Bruker pulse sequences with version 3.2 and patch level 6 TopSpin software (Bruker Daltonics).

\section{In Vitro Experiments}

All incubations and sample analysis were completed according to the following general procedure. Specific details for each experiment are described later. Incubations contained substrate, enzyme, and inhibitor in $100 \mathrm{mM}$ potassium phosphate buffer, $\mathrm{pH} 7.4$, containing $3 \mathrm{mM} \mathrm{MgCl}_{2}$. The solutions were prewarmed to $37^{\circ} \mathrm{C}$ and then the reactions were initiated by adding prewarmed $\mathrm{NADPH}$ solution to $1 \mathrm{mM}$ final concentration. All reactions were conducted at $37^{\circ} \mathrm{C}$ with shaking. At the end of the incubations, reactions were quenched with two volumes of cold acetonitrile containing propranolol $(0.01 \mu \mathrm{M})$ as an internal 
TABLE 1

Synthesized chemical standards that were tested as proposed rearrangement products of GDC-0339 and its desfluoro analog<smiles>[R]c1c(NC(=O)c2nc(-c3c(F)cccc3F)sc2N)cnn1C</smiles>

Where unspecified stereochemistry is shown, individual enantiomers were synthesized and tested.

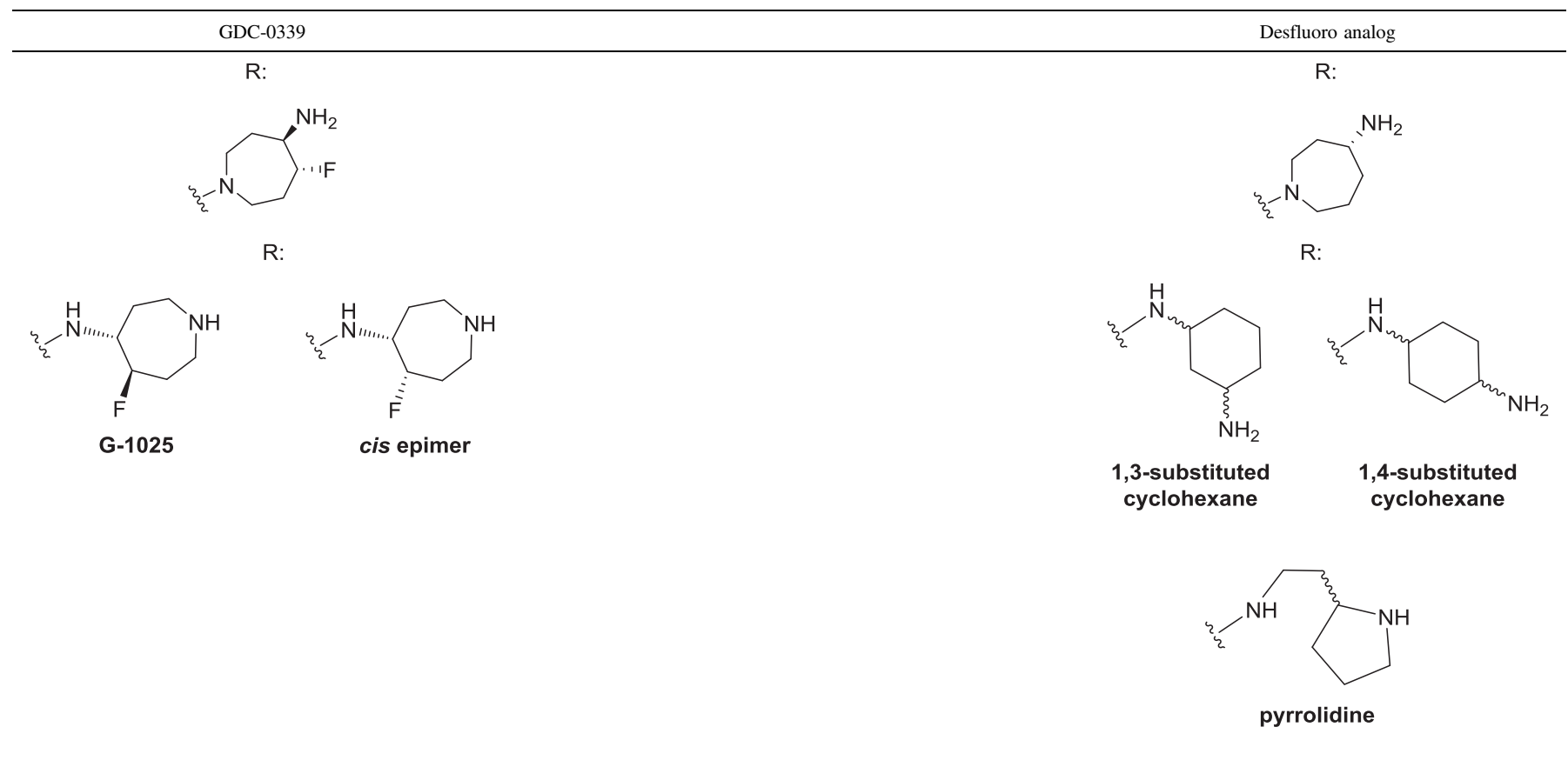

standard. Proteins were precipitated and removed by centrifuging the samples. The supernatants were diluted with an equal volume of water for LC-MS/MS analysis.

In vitro formation of G-1025 from GDC-0339 and acetaminophen from phenacetin were measured using a QTRAP 5500 (AB Sciex, Foster City, CA) mass spectrometer equipped with a CTC PAL autosampler (LEAP Technologies, Carrboro, NC) and 1290 pump (Agilent Technologies, Santa Clara, CA). Analytes were chromatographically separated with a Kinetex $1.7 \mu \mathrm{m}, \mathrm{XB}-\mathrm{C} 182.1 \times$ $50 \mathrm{~mm}$ (Phenomenex) column with a gradient program of $0.1 \%$ formic acid in water and acetonitrile. The total run-time was 2.8 minutes. The SRM transitions monitored were $m / z, 466.3 \rightarrow m / z, 72.0$ for GDC-0339, $\mathrm{m} / \mathrm{z}, 466.3 \rightarrow \mathrm{m} / \mathrm{z}, 116.1$ for G-1025, $\mathrm{m} / \mathrm{z} \quad 152.0 \rightarrow \mathrm{m} / \mathrm{z} 110.0$ for acetaminophen, and $\mathrm{m} / \mathrm{z} 260.0 \rightarrow \mathrm{m} / \mathrm{z}$ 116.0 for propranolol.

Incubations with Cryopreserved Hepatocytes, Liver Microsomes and Recombinant Human P450 Enzymes. The initial profiling of metabolites of GDC-0339 was completed with incubations of suspensions of cryopreserved hepatocytes from rats and humans. Hepatocytes were rapidly thawed and resuspended in Dulbecco modified Eagle's medium at $\sim 1 \times 10^{6}$ cells $/ \mathrm{ml}$. GDC-0339 was added to a final concentration of $5 \mu \mathrm{M}$ and incubated for 3 hours at $37^{\circ} \mathrm{C}$. The reactions were stopped by the addition of acetonitrile and, following centrifugation, the supernatants were profiled by LC-MS.

The formation of G-1025 from GDC-0339 in vitro was tested with rat (not pretreated or $\beta$-naphthoflavone, dexamethasone, or corn oil pretreated) or human liver microsomes $(0.5 \mathrm{mg} / \mathrm{ml})$ or individual recombinant human P450 Supersomes (CYP1A1, 1A2, 2B6, 2C8, 2C9, 2C18, 2C19, 2D6, 2E1, 3A4, and 3A5, $40 \mathrm{pmol} / \mathrm{ml}$ ). GDC-0339 was added to a final $1 \mu \mathrm{M}$ concentration, and samples were incubated for 60 minutes. Triplicate incubations were completed for each P450 isoform. Negative control incubations contained liver microsomes not supplemented with NADPH or vector-only-transfected Supersomes that were supplemented with NADPH. To test the effect of P450 inhibition on product formation, liver microsomes were preincubated with 1-aminobenzotriazole (ABT, $1 \mathrm{mM})$ and NADPH $(1 \mathrm{mM})$ for 15 minutes prior to addition of GDC-0339. Reversibility of the reaction was investigated by incubating G-1025 with liver microsomes and monitoring for GDC-0339 formation. Structural analogs of GDC-0339 (Table 2) were tested if they underwent the same rearrangement reaction as GDC-0339. Each compound was individually incubated with $\beta$-naphthoflavone-pretreated rat liver microsomes supplemented with NADPH in the same manner as was done for GDC-0339 (described above). The resulting incubates were analyzed by LC-MS/MS to assess whether a rearrangement product had formed.

Enzyme Kinetics Studies. Under in vitro conditions determined to be initial rate, formation of G-1025 from GDC-0339 was evaluated. GDC-0339 concentrations ranged from 0.1 to $50 \mu \mathrm{M}$ and recombinant CYP1A1 protein was $3 \mathrm{pmol} / \mathrm{ml}$. Triplicate incubations were completed at each GDC-0339 concentration. Enzyme and substrate were preincubated in buffer for 10 minutes, followed by another 7.5 minutes after the reactions were initiated with the addition of NADPH. The concentrations of G-1025 at the end of the incubations were determined by LC-MS/MS by comparison to calibration solutions prepared from G-1025 synthetic standard. The apparent Michaelis constant $\left(\mathrm{K}_{\mathrm{m}}\right)$ and maximum reaction rate $\left(\mathrm{V}_{\max }\right)$ values were obtained by nonlinear regression fitting with the Michaelis-Menten equation using Prism 4.0 software (GraphPad Software Inc., San Diego, CA). 
TABLE 2

GDC-0339 analogs that were tested as substrates for in vitro rearrangement by incubating with $\beta$-naphthoflavone-pretreated rat liver microsomes<smiles>[R]c1c(NC(=O)c2nc(-c3c(F)cccc3F)sc2N)cnn1C</smiles>

Where stereochemistry is not indicated a racemic mix of enantiomers was tested.

Evidence for rearrangement

Inhibition of CYP1A1 Activities by GDC-0339. The inhibition of CYP1A1mediated phenacetin $O$-deethylation to acetaminophen was characterized for GDC-0339, G-1025, and $\alpha$-naphthoflavone (positive control). Incubation mixtures consisted of CYP1A1 Supersomes $(20 \mathrm{pmol} / \mathrm{ml})$, phenacetin (at final concentrations ranging from 15 to $400 \mu \mathrm{M}$ ), and inhibitors of varying concentrations. GDC-0339 and G-1025 were tested at final concentrations of $0.1-10 \mu \mathrm{M}$ and $\alpha$-naphthoflavone was tested at final concentrations of $0.05-2.5 \mu \mathrm{M}$. The inhibitor stock solutions were made up in dimethyl sulfoxide (DMSO); therefore, all incubations (with and without inhibitors) were made to contain $0.1 \%$ DMSO by volume. Reactions were conducted for 10 minutes. The final volume of reaction solution was $150 \mu \mathrm{l}$. Triplicate incubations were conducted at each substrate and inhibitor concentration. The concentrations of acetaminophen at the end of the incubations were determined by LC-MS/MS by comparison to calibration solutions prepared from acetaminophen standard. No mode of inhibition was assumed and the reaction kinetics for the full data set of substrate and inhibitor concentrations was fit by nonlinear regression analysis with a mixed mode of inhibition. Lineweaver-Burk plots were constructed to visualize the kinetic data and fit by linear regression. The changes in slope and $y$-intercept associated with varying inhibitor concentrations were inspected to judge the mode of inhibition. All data fitting and parameter estimates were completed using Prism 4.0 software (GraphPad).

\section{Results}

Identification of the Rearranged Metabolite. In rat and human hepatocyte incubations, the major metabolic pathways for GDC-0339 were identified as glucuronidation, pyrazole $N$-demethylation, hydroxylation at aminoazepane, and azepane $N$-dealkylation to 1-methyl-pyrazol-5amine on the basis of the UV profile of metabolites generated. G-1025 was generated as a minor metabolite (i.e., not detected by UV but detected by mass spectrometry) and it was the focus of further studies given its nonobvious structure and reaction mechanism of formation. In the high-resolution full scan, protonated molecular ion $[\mathrm{M}+\mathrm{H}]^{+}$was observed at $m / z, 466.1617\left(\mathrm{C}_{20} \mathrm{H}_{23} \mathrm{~F}_{3} \mathrm{~N}_{7} \mathrm{OS}^{+},-2.9 \mathrm{ppm}\right)$, which was identical to the observed protonated molecular ion for GDC-0339 (Fig. 1). The cis-diastereomer of GDC-0339 $((4 R, 5 S)$ or $(4 S, 5 R)-4$ amino-5-fluoroazepane) was also observed in the incubation samples but it was chromatographically separated from GDC-0339 and the rearrangement product. In the data-dependent MS/MS experiments, the fragment ion pattern of the rearrangement metabolite was distinct from GDC-0339. For GDC-0339, the abundant product ions were observed at $m / z 449$ (loss of $\mathrm{NH}_{3}$ ), $\mathrm{m} / \mathrm{z} 446$ (loss of HF), and $\mathrm{m} / \mathrm{z}$ 429 (loss of $\mathrm{NH}_{3}$ and $\mathrm{HF}$ ) (Fig. 2A). In comparison, the rearranged product was observed with product ions at $\mathrm{m} / z 446$ (loss of HF) and $m / z 351$ (loss of $\mathrm{C}_{6} \mathrm{H}_{10} \mathrm{NF}$ ) (Fig. 2B). These ions suggested the majority the molecule was unchanged. The ion at $\mathrm{m} / \mathrm{z}, 351$ by fragmentation at azepane was highly favored for G-1025 but not for GDC-0339, which suggested that azepane had been modified. Further, the absence of the ion at $m / z, 449$ for G-1025 indicated that the primary 

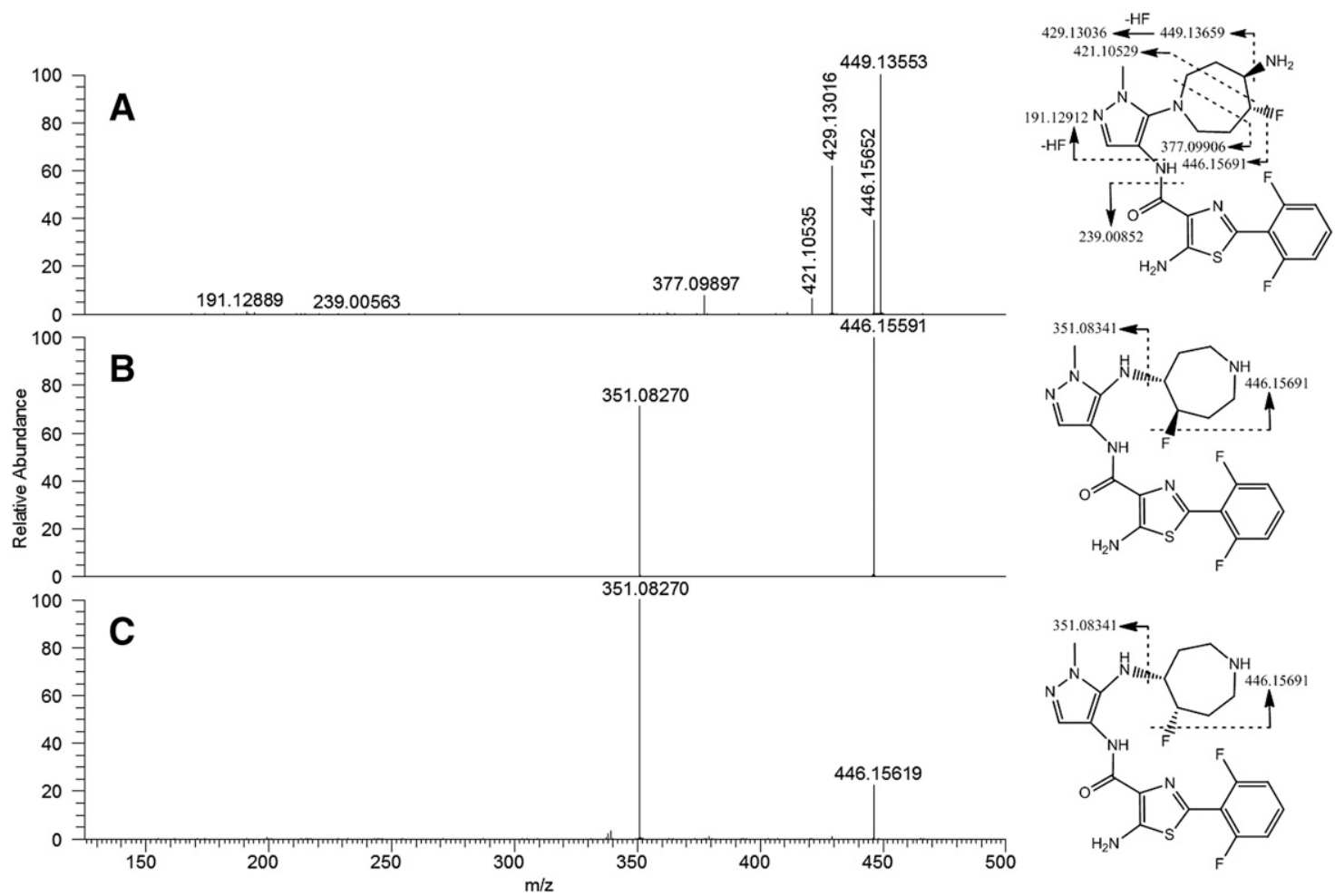

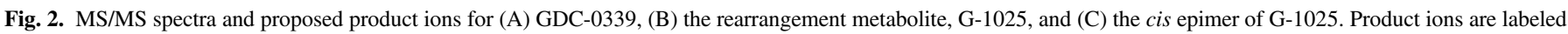
with calculated $\mathrm{m} / \mathrm{z}$ values.

amine of GDC-0339 had been stabilized, which further supported changes at azepane and its attachment to the pyrazole. However, there were no diagnostic ions that identified the new structure or its new connectivity.

Structure Elucidation of the Rearrangement Product. Early metabolite structure elucidation experiments using the desfluoro analog of GDC-0339 ( 2 in Table 2) compared its rearrangement product with synthetic standards for its proposed products, 1,3- and 1,4-substituted cyclohexane or pyrrolidine (Table 1). In each case, chromatographic elution and MS/MS spectra of the metabolite differed from the standard.

The rearrangement metabolite of GDC-0339 was isolated from rat urine using preparative liquid chromatography and fraction collection. In NMR experiments, proton and carbon chemical shifts observed for the isolated metabolite were in agreement with those of GDC-0339. ${ }^{1} \mathrm{H}-{ }^{13} \mathrm{C}$ heteronuclear multiple-bond correlations $(\mathrm{HMBC})$ were used to confirm that the alterations had been made for attachment of the azepane ring to the pyrazole. Key correlations NH-17 to C-22, H-23 to C-22, and H-19 to $\mathrm{C}-22$ proved that the azepane was still attached in the same location at GDC-0339. However, new correlations from NH-24 to C-18 and NH-24 to $\mathrm{C}-22$ proved that the azepane was now attached through a secondary amine rather than the azepane nitrogen in GDC-0339 (Fig. 3). Additional NMR data are provided in Supplemental Table 2 and Supplemental Figs. 1-5.

With the structural information provided by NMR analysis of the isolated metabolite, chemical standards for G-1025 (5-amino-2(2,6-difluorophenyl)- $N$-(5-((5-fluoroazepan-4-yl)amino)-1-methyl1H-pyrazol-4-yl)thiazole-4-carboxamide, a mixture of two enantiomers as trans 4-amino-5-fluoroazepane) and its cis epimer (a mixture of two enantiomers) were synthesized (Table 1). Chromatographic separation of the epimers was accomplished (data not shown). Also, relative abundances of the product ions were observed to differ between the two epimers (Fig. 2, B and C). The rearrangement metabolite matched
G-1025, and not the cis epimer, in mixed sample experiments as demonstrated by coelution with the in vivo metabolite in urine and matching MS/MS spectra. This confirmed the metabolite structure and that the trans configuration of amino and fluorine substituents at azepane had been retained.

Rearrangement of GDC-0339 Structural Analogs. A series of compounds that modified the aminoazepane but otherwise retained the GDC-0339 structure (Table 2) were individually incubated with $\beta$-naphthoflavone-pretreated rat liver microsomes. This in vitro system was selected because it provided the most extensive formation of G-1025 from GDC-0339. Each of the rearrangement products showed an MS/MS spectrum that was very similar to that of G-1025, with an abundant product ion at $m / z 351$, which was rationalized as 1-methyl-1H-pyrazol-5-aminium (Fig. 2). If the rearrangement metabolite was detected by MS in the in vitro sample, the compound was categorized as undergoing the reaction (Table 2).

In Vitro Formation of the G-1025. Incubations of GDC-0339 with liver microsomes generated the rearrangement product only when supplemented with NADPH. By relative comparison of mass spectral signals normalized to the internal standard, G-1025 formation was more extensive by rat liver microsomes than by human, and in rats, $\beta$-naphthoflavone pretreatment enhanced the metabolite generation compared with dexamethasone or vehicle pretreatment (data not shown). In addition, preincubation of microsomes with ABT, a panP450 mechanism-based inactivator, abolished G-1025 formation. After incubating GDC-0339 with individual recombinant human P450s, the rearrangement metabolite of GDC-0339 was most extensively formed by CYP1A1 (Fig. 4). No reversibility of the reaction was observed, since GDC-0339 was not formed in incubations of G-1025 with liver microsomes. Saturation of G-1025 formation by singly expressed recombinant CYP1A1 was achieved by increasing GDC-0339 initial concentrations and allowed estimates of the kinetic parameters. The 


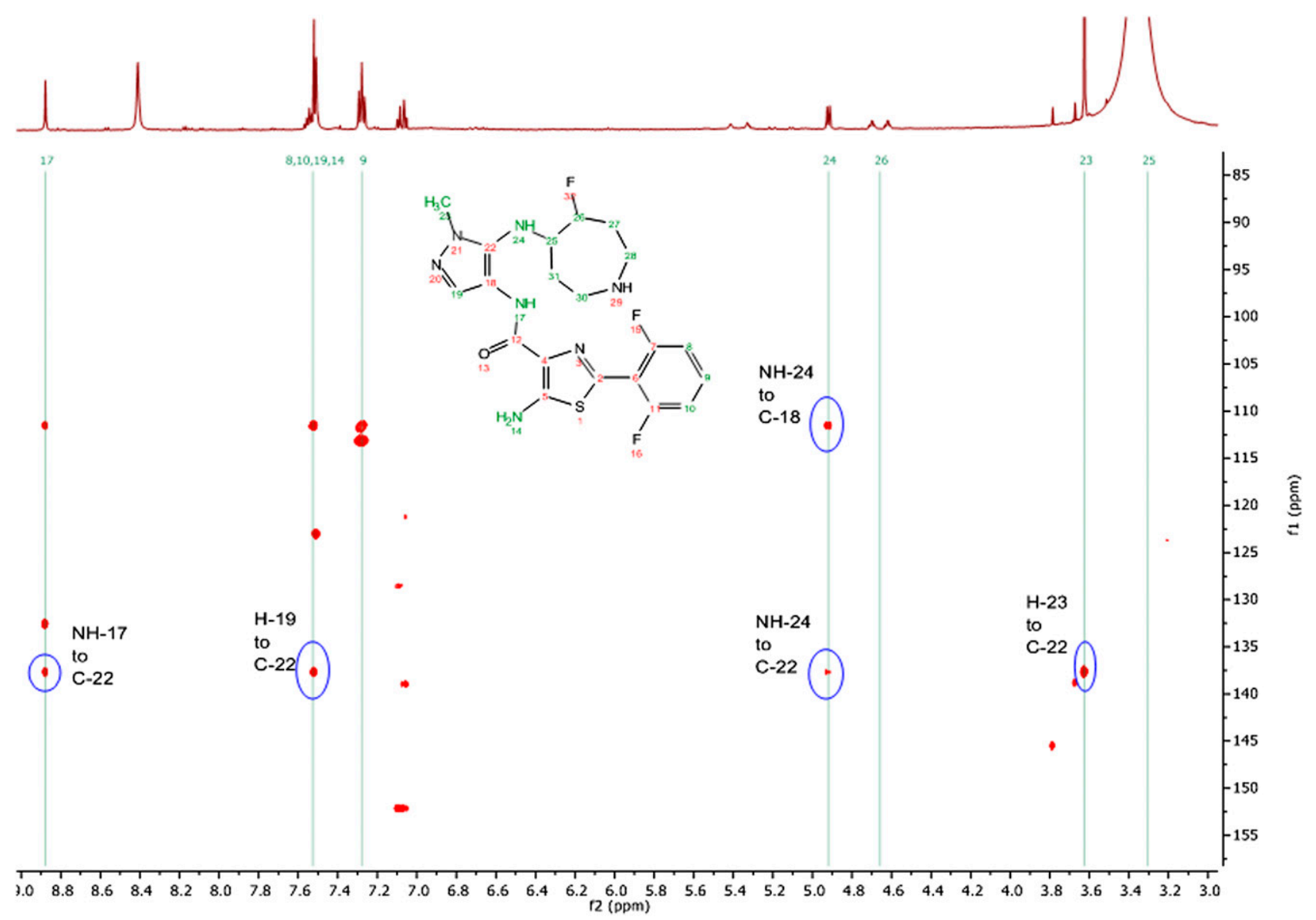

Fig. 3. ${ }^{1} \mathrm{H} /{ }^{13} \mathrm{C}$ heteronuclear multiple-bond correlation spectrum of the rearrangement metabolite of GDC-0339 isolated from rat urine. Key correlations used for the structure determination are in circles with their assignments labeled.

reaction was best described by typical Michaelis-Menten kinetics, and nonlinear regression fitting provided estimates for apparent $\mathrm{k}_{\mathrm{cat}}$ and $\mathrm{K}_{\mathrm{m}}$ as $8.4 \pm 0.4$ minutes $^{-1}$ and $0.6 \pm 0.1 \mu \mathrm{M}$, respectively.

Direct CYP1A1 Inhibition by GDC-0339. The Michaelis-Menten plots for phenacetin $O$-deethylation to acetaminophen by CYP1A1 Supersomes with increasing concentration of GDC-0339 are shown in Fig. 5A. Phenacetin formation rates in absence of GDC-0339 followed typical saturation kinetics described by estimates of apparent turnover number $\left(\mathrm{k}_{\mathrm{cat}}\right)$ and $\mathrm{K}_{\mathrm{m}}$ of $21.5 \pm 0.6$ minutes $^{-1}$ and $26 \pm 3 \mu \mathrm{M}$, respectively. When GDC-0339 was added up to $1 \mu \mathrm{M}$, similar maximal reaction rates were reached but required higher phenacetin concentrations, and the apparent $K_{m}$ increased with increasing GDC-0339. When GDC- 0339 was added at 5 and $10 \mu \mathrm{M}$, the maximal reaction rates were not reached at $400 \mu \mathrm{M}$ phenacetin. Lineweaver-Burk plots of the transformed kinetics data were generated for visual inspection and are presented in Fig. 5B. With GDC-0339 addition, the slopes were impacted, but $y$-intercepts were not, which identified the mode of inhibition to be predominantly competitive. The inhibition constant $\left(\mathrm{K}_{\mathrm{i}}\right)$ for GDC-0339 was estimated to be $0.9 \pm 0.2 \mu \mathrm{M}$. In contrast, G-1025 did not effectively inhibit phenacetin $O$-deethylation. In the same set of experiments, $\alpha$-naphthoflavone was characterized as a competitive inhibitor with an estimated $\mathrm{K}_{\mathrm{i}}$ of $2.6 \pm 1.2 \mu \mathrm{M}$.

\section{Discussion}

The in vitro and in vivo metabolite profiling for GDC-0339 identified a rearrangement metabolite. This metabolite was observed in rat samples, including plasma, and experiments with cryopreserved hepatocytes and liver microsomes indicated that it could also be formed in humans. Though a 10 - to 20 -fold reduction in binding potency of the rearranged metabolite compared with GDC-0339 was expected on the basis of the activity comparison of an analogous pair (compound 1 and its rearranged metabolite, Supplemental Fig. 6), further work on this metabolite was warranted. This metabolite and its formation were notable given that no change in redox state was evident. Further, the rearrangement product was found in vivo in rats, which necessitated its consideration for bioanalytical method selectivity, since it and GDC0339 would not be easily differentiated by mass spectrometric detection. Though the profiling experiments showed they were separated with chromatography, the extended analysis time could be a detriment to bioanalysis sample throughput.

MS experiments of GDC-0339 and the rearrangement product revealed some clear differences in their fragmentation behaviors. This ruled out that the rearrangement was a simple change in stereochemistry. The abundant ion at $\mathrm{m} / \mathrm{z}, 351$ confirmed retention of a $1 \mathrm{H}$-pyrazol-5amine connection of azepane. The strong intensity for this ion with G-1025 compared with GDC-0339 suggested a modification of the tertiary amine of the aminoazepane. Also, the loss of ammonia was not observed for G-1025, which suggested the amino moiety as another site of modification. However, no other product ions were generated that provided insight into how the azepane or its connectivity had been modified. Our initial proposal for the rearrangement was azepane-opening via P450-mediated $N$-dealkylation, then subsequent ring-closing and reduction to result in an altered ring size. A 


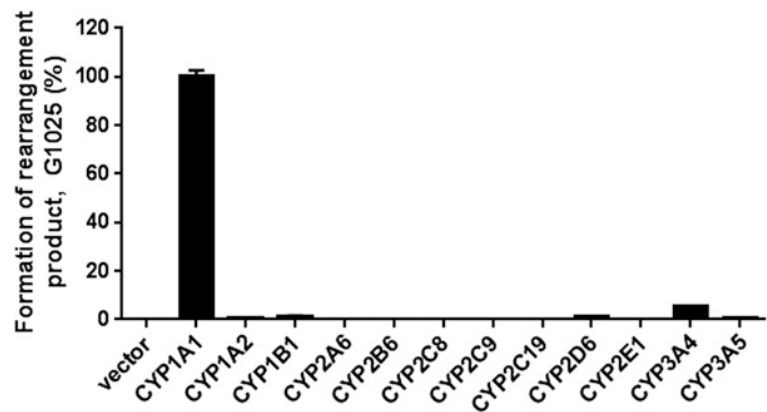

Fig. 4. Formation of the rearrangement metabolite, G-1025, in incubations of GDC0339 with individual recombinant human P450 isozymes. Incubations contained equal P450 content. The extent of G-1025 formations for each tested enzyme was normalized to CYP1A1, which showed the greatest metabolite formation and was set as $100 \%$. Columns and error bars represent the mean and S.D. of triplicate incubations.

similar mechanism was enacted to rationalize the biotransformation of aminopyrrolidine to aminopiperidine in AMG657417 (Chen et al., 2011). With this mechanism in mind, several ring closure possibilities were considered and tested with 1,3- and 1,4-substituted cyclohexane and pyrrolidine synthetic standards (Table 1). However, none of these standards matched the rearranged metabolite. We also considered oxidation of the primary amine, which could activate a ring expansion reaction and result in 1,5-diazocane; this being analogous to cyclopropyl expansion to cyclobutyl (Bondon et al., 1989). However, these predicted metabolite structures were not correct. To probe the reaction, we investigated in vitro whether an exogenous primary amine (lysine or cycloheptylamine added in molar excess) would replace azepane and whether an intermediate could be trapped with glutathione, methoxylamine, or (2,2,6,6-tetramethylpiperidin-1-yl)oxyl (TEMPO), but these experiments did not provide any additional insight into how GDC-0339 was being activated for the rearrangement.

Since our efforts to confirm plausible structures were ineffective, we pursued defining the structure directly. The metabolite was isolated by preparative high-performance liquid chromatography of urine collected from rat in vivo studies of GDC-0339 in sufficient quantity and purity for NMR characterization. These data were consistent with the new connectivity of azepane to pyrazole being through a secondary amine. Two diastereoisomers of trans- and cis-configurations were synthesized. Coinjection of the synthetic standard with in vitro and in vivo samples in the $\mathrm{MS}^{\mathrm{n}}$ experiments confirmed the rearranged product to be G-1025 and that the trans stereochemistry was maintained during the rearrangement.

To rationalize the observations, we propose a radical mechanism for the rearrangement, which is presented in Fig. 6. P450 initiates the reaction by abstracting a hydrogen atom from the exposed primary amine. This nitrogen-centered radical (intermediate I) can then combine with the pyrazole and transfer the radical to a more stable aromatic ring (II). This step suggests sufficient flexibility in the aliphatic ring to bring these sites in proximity of each other to react. Bond-forming and bondbreaking via single electron transfers (III) results in exchange of nitrogen bonded to pyrazole such that the tertiary azepane amine and primary amine of GDC-0339 are both transformed to secondary amines in G-1025 (IV). The reaction would then be quenched with a hydrogen donation to the azepane radical.

To provide a more complete description of the reaction, we completed in vitro experiments to understand the enzymology. The rearrangement product was formed by liver microsomes from rats and humans. The reaction required NADPH and was effectively inhibited by ABT, which are consistent with the involvement of $\mathrm{P} 450$ enzymes. The identity of the P450s capable of catalyzing the reaction was defined by incubating
A
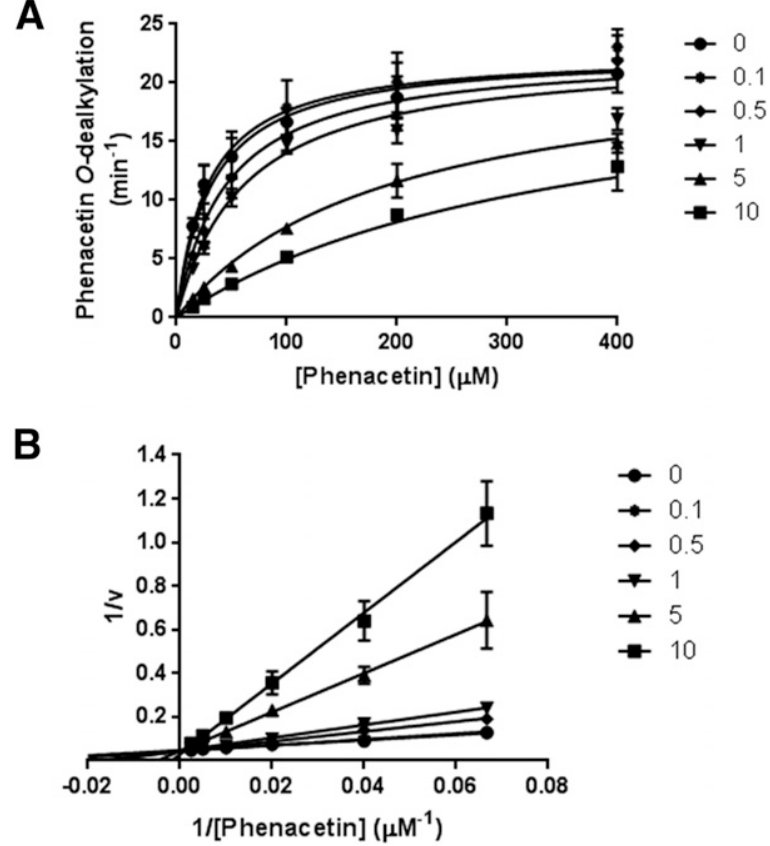

Fig. 5. Reaction kinetics for phenacetin $O$-deethylation to acetaminophen by CYP1A1 Supersomes with direct inhibition by GDC-0339 (A) and transformed kinetic data in Lineweaver-Burk plots used to judge the mode of inhibition to be predominantly competitive (B). Different tested concentrations for GDC-0339 are represented as different symbol shapes as described in legend on the right. Data points and error bars represent mean and S.D. of triplicate incubations.

GDC-0339 with a panel of singly expressed human P450s. High P450isoform selectivity was observed with CYP1A1 efficiently catalyzing this reaction. This was further supported by higher G-1025 formation using liver microsomes from rats pretreated with $\beta$-naphthoflavone. In contrast, no increases in G-1025 formation were observed for dexamethasone-pretreated rat liver microsomes, which suggested a minor role for CYP3A enzymes. Kinetic analysis revealed the CYP1A1-mediated reaction to be high affinity (apparent $\mathrm{K}_{\mathrm{m}} \sim 1 \mu \mathrm{M}$ ) with turnover $\left(\mathrm{k}_{\mathrm{cat}}\right)$ of 8.4 minutes $^{-1}$.

The binding of GDC-0339 at the active site of CYP1A1 was confirmed by its direct inhibition of phenacetin $O$-deethylation. Phenacetin is a known substrate of CYP1A enzymes, though it has somewhat greater selectivity for CYP1A2 compared with CYP1A1 (Tassaneeyakul et al., 1993; Huang et al., 2012). With docking studies using the solved structure for CYP1A1, phenacetin orients in the active site with its ethoxy group appropriately placed in proximity at the active site heme for oxidation (Walsh et al., 2013). Using phenacetin $O$-deethylation by CYP1A1 as the object reaction for inhibition, GDC-0339 was observed to have $\sim 3$-fold greater potency than $\alpha$-naphthoflavone, whereas G-1025 was not a potent inhibitor. The mode of inhibition by GDC-0339 was principally competitive, as evidenced by large changes in slopes but not in $y$-intercepts in the Lineweaver-Burk plots. Competitive enzyme inhibition requires binding of inhibitor to the active site on the enzyme to prevent substrate binding. Thus, these data were indicative of GDC-0339 binding at the active site of CYP1A1.

CYP1A1 is predominantly expressed extrahepatically though large interindividual variation in its levels has been reported. From a panel of human intestinal microsomes, CYP1A1 was detected by immunoblotting in 3 of 31 donors (Paine et al., 2006). In liver, CYP1A1 mRNA was found in approximately half of the tested human liver samples, and expression was much lower than that for closely related CYP1A2 


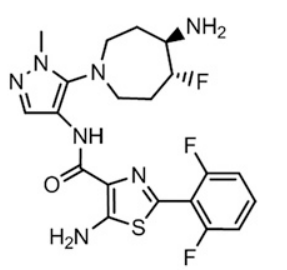

GDC-0339

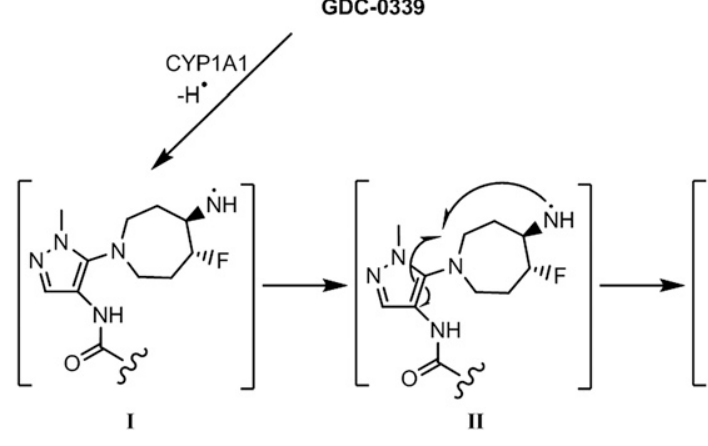

I

II

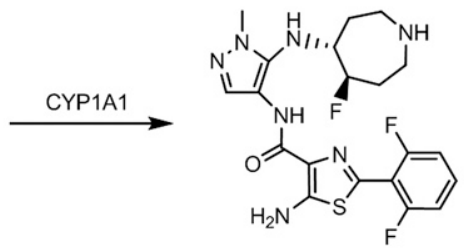

G-1025

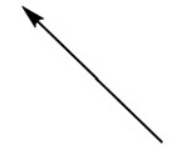

Fig. 6. Proposed mechanism for the CYP1A1mediated rearrangement reaction of GDC-0339 to $\mathrm{G}-1025$.

(McKinnon et al., 1991). This high interindividual variability may result from its expression being inducible (Kashfi et al., 1995). The protein retains a high level of sequence conservation between humans and rats (83\% amino acid sequence) and, as in humans, it is found at low levels in rat liver and predominantly expressed extrahepatically (Martignoni et al., 2006). Though CYP1A1 is expressed at low levels in livers of rats and humans, its efficiency in catalyzing the reaction was very high. Thus, it is difficult to clearly define the contribution of CYP1A1 versus other P450s to the total formation of G-1025 in liver microsomes or in vivo. Other P450s, such as CYP3A4 in humans, were much less efficient catalysts for the rearrangement but are expressed at much higher levels, and potentially have significant contributions to G-1025 formation.

The human CYP1A1 structure has been solved, which provides insight into its role in metabolizing xenobiotics (Walsh et al., 2013). The structure with $\alpha$-naphthoflavone bound showed a narrow, enclosed active site, and this was consistent with its recognized roles in metabolizing polycyclic aromatic hydrocarbons and other planar molecules. In contrast, the aminoazepane of GDC-0339 that is metabolized by CYP1A1 is a flexible, nonplanar structure that may require conformational changes by the enzyme to allow it to bind. Further, if subsequent steps of the intramolecular concerted rearrangement occur within the active site, in order to accommodate our proposed mechanism of aminoazepane folding upon itself, the enzyme would require substantial flexibility to facilitate the diamine intermediate (III in Fig. 6). Both of these descriptors of the active site were unexpected given the previously described reactions for CYP1A1.

A partial structure-activity analysis for this rearrangement reaction was compiled by testing compounds structurally similar to GDC-0339 that were synthesized during the lead optimization efforts that have been previously described (Wang et al., 2015). When incubated with induced rat liver microsomes, a number of these compounds showed formation of rearrangement metabolites. On the basis of these results, we categorized substrates as able to undergo the rearrangement reaction or not (Table 2 ). The absolute stereochemistry of the primary amine $(1,2)$ and its position at the azepane ring (1-3) didn't impact the rearrangement biotransformation. Cis isomer of GDC-0339 (4) also yielded the rearranged metabolite. Substitutions at $\beta$ or $\gamma$ position of the primary amine ( 5 or 6 ) were well tolerated. Additionally, the size and flexibility of azepane was not a necessity, since smaller rings, 3-aminopiperidine (7) or 3-aminopyrrolidine (8), also underwent the rearrangement. However, methyl substitution at the $\alpha$ carbon to the primary amine (9) abolished the rearrangement reaction, implying that steric hindrance around the basic amine matters, which was consistent with our hypothesized mechanism of action. In addition, taking away the postulated reaction centers, such as replacing the primary amine with a hydroxyl group (10) or substituting the nitrogen in the azepane with a carbon (11) both resulted in a shutdown of the rearrangement reaction. Again, the structure-activity relationship corroborated our proposed mechanism. There is some indication that, depending on substrate structure, other P450s are capable of activating the amino group for this reaction. For the desfluoro analog of GDC-0339 (2), preliminary P450 phenotyping indicated CYP1A1 most efficiently catalyzed the rearrangement reaction, but CYP2D6 and CYP3A4 had much higher activities compared with those observed for GDC-0339. Our work in this area will continue with testing how physicochemical properties of the substrate influence the rates or enzymes involved for the rearrangement reaction. Such analyses have been made for fluorinated analogs of propranolol and benzphetamines, which showed increasing interactions with CYP2D6 and CYP2B4, respectively, with decreasing pKa (Upthagrove and Nelson, 2001; Sheng et al., 2009).

In summary, an unexpected and unique metabolite was observed for GDC-0339 as a result of an intramolecular rearrangement reaction catalyzed by $\mathrm{P} 450 \mathrm{~s}$. We propose that the reaction is initiated by hydrogen abstraction from the primary amine, which then initiates bond forming and bond breaking through radical reactions. Interestingly, the in vitro reaction was most efficiently catalyzed by human CYP1A1, which demonstrates a ligand for CYP1A1 that does not conform to descriptors of its known substrates.

\section{Acknowledgments}

We thank Dr. Emily Scott (University of Michigan) for helpful discussion of findings from the present investigation.

\section{Authorship Contributions}

Participated in research design: Takahashi, X. Wang, Segraves, J. Wang, Ma.

Conducted experiments: Takahashi, Segraves, J. Wang, Ma.

Contributed new reagents or analytic tools: $\mathrm{X}$. Wang.

Performed data analysis: Takahashi, Segraves, Ma.

Wrote or contributed to the writing of the manuscript: Takahashi, X. Wang, Segraves, Chang, Khojasteh, Ma. 


\section{References}

Bondon A, Macdonald TL, Harris TM, and Guengerich FP (1989) Oxidation of cycloalkylamines by cytochrome P-450. Mechanism-based inactivation, adduct formation, ring expansion, and nitrone formation. J Biol Chem 264:1988-1997.

Chen Y, Skiles GL, Shou M, Hickman D, and Hsieh F (2011) A novel biotransformation of alkyl aminopyrrolidine to aminopiperidine ring by human CYP3A. Drug Metab Dispos 39 $1668-1673$.

Guengerich FP and Munro AW (2013) Unusual cytochrome p450 enzymes and reactions. J Biol Chem 288:17065-17073.

Hodges AJ, Matteucci M, Sharpe A, Sun M, Tsui VH, and Wang X (2013) inventors, F. Hoffmann-La Roche Ag, assignee. Pyrazol-4-Yl-heterocyclyl-carboxamide compounds as Pim kinase inhibitors and their preparation. U.S. patent 8,614,206 B2. 2013 Apr 4.

Huang Q, Deshmukh RS, Ericksen SS, Tu Y, and Szklarz GD (2012) Preferred binding orientations of phenacetin in CYP1A1 and CYP1A2 are associated with isoform-selective metabolism. Drug Metab Dispos 40:2324-2331.

Kashfi K, McDougall CJ, and Dannenberg AJ (1995) Comparative effects of omeprazole on xenobiotic metabolizing enzymes in the rat and human. Clin Pharmacol Ther 58:625-630.

Keane NA, Reidy M, Natoni A, Raab MS, and O'Dwyer M (2015) Targeting the Pim kinases in multiple myeloma. Blood Cancer J 5:e325.

Martignoni M, Groothuis GM, and de Kanter R (2006) Species differences between mouse, rat dog, monkey and human CYP-mediated drug metabolism, inhibition and induction. Expert Opin Drug Metab Toxicol 2:875-894.

McKinnon RA, Hall PD, Quattrochi LC, Tukey RH, and McManus ME (1991) Localization of CYP1A1 and CYP1A2 messenger RNA in normal human liver and in hepatocellular carcinoma by in situ hybridization. Hepatology 14:848-856.
Paine MF, Hart HL, Ludington SS, Haining RL, Rettie AE, and Zeldin DC (2006) The human intestinal cytochrome P450 "pie". Drug Metab Dispos 34:880-886.

Sheng X, Zhang H, Im SC, Horner JH, Waskell L, Hollenberg PF, and Newcomb M (2009) Kinetics of oxidation of benzphetamine by compounds I of cytochrome P450 2B4 and its mutants. J Am Chem Soc 131:2971-2976.

Shinkyo R, Xu L, Tallman KA, Cheng Q, Porter NA, and Guengerich FP (2011) Conversion of 7-dehydrocholesterol to 7-ketocholesterol is catalyzed by human cytochrome P450 7A1 and occurs by direct oxidation without an epoxide intermediate. J Biol Chem 286:33021-33028.

Tassaneeyakul W, Birkett DJ, Veronese ME, McManus ME, Tukey RH, Quattrochi LC, Gelboin HV, and Miners JO (1993) Specificity of substrate and inhibitor probes for human cytochromes P450 1A1 and 1A2. J Pharmacol Exp Ther 265:401-407.

Upthagrove AL and Nelson WL (2001) Importance of amine pKa and distribution coefficient in the metabolism of fluorinated propranolol derivatives. Preparation, identification of metabolite regioisomers, and metabolism by CYP2D6. Drug Metab Dispos 29:1377-1388.

Walsh AA, Szklarz GD, and Scott EE (2013) Human cytochrome P450 1A1 structure and utility in understanding drug and xenobiotic metabolism. J Biol Chem 288:12932-12943.

Wang X, Sun M, New C, Nam S, Blackaby WP, Hodges AJ, Nash D, Matteucci M, Lyssikatos JP, Fan PW, et al. (2015) Probing mechanisms of CYP3A time-dependent inhibition using a truncated model system. ACS Med Chem Lett 6:925-929.

Address correspondence to: Dr. Ryan H. Takahashi, Drug Metabolism and Pharmacokinetics, Genentech, Inc., 1 DNA Way, MS 412a, South San Francisco, CA 94080. E-mail takahashi.ryan@gene.com 